\title{
Microwave-acceleration of Carboxamides Formation Using Water Soluble Condensing Agent DMT-MM or DCC ${ }^{\mathrm{a}}$
}

\author{
Nam Sook Cho*, Hye Jin Jeon, and Dong Uk Heo \\ Department of Chemistry, Chungnam National University, Daejeon 305-764, Korea \\ *E-mail: nsmcho@cnu.ac.kr
}

(Received May 7, 2012; Accepted July 20, 2012)

Key words: Amide, DCC, 4-(4,6-Dimethoxy-13,5-triazin-2-yl)-4-methylmorpholinium chloride, Dicyclohexylcarbodiimide, Microwave

Amide linkages are present in key natural products and in many biologically active molecules. Therefore, the formation of amide bonds is important to both synthetic chemists and biologists. Approximately $66 \%$ of all preliminary screening reactions in industrial medicinal chemistry laboratories involve amide formation. ${ }^{1}$ Although many methods have been developed for amide synthesis, a procedure for the direct coupling of amines with an acid in the presence of coupling agents without prior activation would be useful for building small-molecule libraries and for key steps in total syntheses. ${ }^{2}$ Condensing agents such as carbodiimides ${ }^{3}$ or other activating agents ${ }^{4-12}$ are generally employed under dry conditions. However, the condensing agent 4-(4,6-dimethoxy-1,3,5-triazin-2-yl)-4methylmorpholium chloride (DMT-MM) can be used to prepare carboxamides in alcohol or water. ${ }^{13-14}$ This reaction is technically simple and relatively easy to implement, requiring only a mixing of acids, amines, and DMT$\mathrm{MM}$ in $\mathrm{MeOH}$ or $\mathrm{H}_{2} \mathrm{O}$. The synthesis of DMT-MM is also relatively simple, and it can be prepared from CDMT (2chloro-4,6 dimethoxy-1,3,5-triazine) and N-metheylmorphorine (NMM) in THF. ${ }^{15}$

The current report describes the application of microwave-induced acceleration to further improve the reaction conditions for amide synthesis using DMT-MM. Highspeed synthesis using microwave technology has attracted considerable attention in organic chemistry. ${ }^{16-18}$ Microwave irradiation provides advantages over conventional heating for chemical transformations, including accelerated reaction rates (and consequently reduced reaction times), higher yields, and cleaner reactions. ${ }^{18}$ These advantages led us to evaluate the use of microwave irradiation for amidation reactions involving less reactive secondary

$\overline{{ }^{a}}$ This research was performed by undergraduate student's experimental project and primary amines with acid in the presence of DMT-MM. We also compared the effectiveness of dicyclohexylcarbodiimide (DCC), a well-known condensing reagent, ${ }^{3}$ when coupled with microwave irradiation versus classical thermal methods.

The reactions were performed by dissolving amines, carboxylic acids, and the condensing agent in solvent, followed by irradiation in a temperature-controlled microwave oven. For comparison, the same reactions were carried out under conventional heating conditions. The amidation of 2-phenylethylamine (a primary amine) with substituted benzoic acid in the presence of DMT-MM in $\mathrm{MeOH}$ gave a yield of over $90 \%$ products within $25 \mathrm{~min}$ at $110{ }^{\circ} \mathrm{C}$ with microwave irradiation. The reaction time with conventional heating was seven times that with microwave irradiation, and the yield was less than $10-20 \%$ of the yield with the microwave-assisted method. The reaction of piperidine (a secondary amine) with benzoic acid afforded a $91 \%$ yield within $30 \mathrm{~min}$ at $150^{\circ} \mathrm{C}$ with microwave irradiation; note the higher reaction temperature and longer reaction time compared with those of the primary amine reaction.

Amidation of piperidine with substituted benzoic acid in the presence of DCC with pyridine in THF yielded more than $60 \%$ products within $25 \mathrm{~min}$ at $120{ }^{\circ} \mathrm{C}$ with microwave irradiation. Thus, DMT-MM was a much more effective coupling agent than DCC. Further increases in reaction temperature were limited by the potential of solvents for producing an explosive atmosphere within the micro-

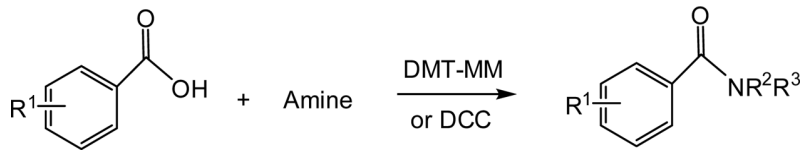

Scheme 1. Preparation of amides using condensing agent DMT$\mathrm{MM}$ or DCC. 
Table 1. Microwave-assisted Formation of Carboxamides Using Condensing Agent DMT-MM or DCC

\begin{tabular}{|c|c|c|c|c|c|c|c|}
\hline \multirow{2}{*}{ Acid } & \multirow{2}{*}{ Amine } & \multicolumn{3}{|c|}{ Microwave irradiation } & \multicolumn{3}{|c|}{ Conventional thermal heating (oil bath) } \\
\hline & & Time (min.) & Temp. $\left({ }^{\circ} \mathrm{C}\right)(\mathrm{W})$ & Yield (\%) & Time (hr.) & Temp. $\left({ }^{\circ} \mathrm{C}\right)$ & Yield $(\%)$ \\
\hline${\text { Benzoic } \text { acid }^{1}}$ & 2-phenylethylamine & 25 & $110(55 \mathrm{~W})$ & 99.0 & 3.5 & Reflux & 88.9 \\
\hline$P$-Nitrobenzoic acid ${ }^{1}$ & 2-phenylethylamine & 25 & $110(55 \mathrm{~W})$ & 92.6 & 3.0 & Reflux & 81.5 \\
\hline$P$-Methoxybenzoic acid ${ }^{1}$ & 2-phenylethylamine & 25 & $110(55 \mathrm{~W})$ & 91.7 & 3.0 & Reflux & 70.8 \\
\hline$m$-Methoxybenzoic acid ${ }^{1}$ & 2-phenylethylamine & 25 & $110(55 \mathrm{~W})$ & 96.0 & 3.0 & Reflux & 72.0 \\
\hline Benzoic acid ${ }^{1}$ & Piperidine & 30 & $150(70 \mathrm{~W})$ & 91.7 & 11.5 & Reflux & 79.3 \\
\hline$P$-Nitrobenzoic acid ${ }^{1}$ & Piperidine & 30 & $150(70 \mathrm{~W})$ & 75.6 & 24.0 & Reflux & 59.6 \\
\hline Benzoic acid ${ }^{2}$ & Piperidine & 25 & $120(60 \mathrm{~W})$ & 63.0 & 10.0 & Reflux & 52.8 \\
\hline$P$-Methoxybenzoic acid ${ }^{2}$ & Piperidine & 25 & $120(60 \mathrm{~W})$ & 60.0 & 12.0 & Reflux & 50.4 \\
\hline
\end{tabular}

${ }^{1}$ Reaction with DMT-MM, solvent MeOH. ${ }^{2}$ Reaction with DCC, solvent THF.

wave oven. Nevertheless, amide formation in THF at a low temperature $\left(120^{\circ} \mathrm{C}\right)$ still reached a yield of $60 \%$ within 25 min with microwave irradiation. Using conventional heating conditions, even with reaction times of more than 40- to 48-fold those used in the microwave experiments, the product yields were much lower (10$16 \%$ ) than those obtained with the application of microwave irradiation.

\section{EXPERIMENTAL}

All ${ }^{1} \mathrm{H}$ NMR spectra were recorded on a Jeol $400 \mathrm{MHz}$ Spectrometer and chemical shifts were recorded to tetramethylsilane (TMS) as an internal standard. Microwaveassistant reactions were performed with an initiator instrument (EXP EU, Biotage, 400W, $2450 \mathrm{MHz}$ ) Each microwave irradiation reaction was carried out in a $5 \mathrm{~mm}$ thickness Biotage vial sealed with a crimp cap. Reaction temperature were measured using infrared sensors on the outer surface of the traction vil. Products were purified by flash chromatography on 200-400 mesh ASTM 60 silica gel.

General method of formation of amides from substituted benzoic acid and 2-phenyethylamine or piperidine in presence of DMT-MM under microwave irradiation Acid (1.5 mmol), 2-phenylethylamine (1.65 mmol, $200 \mathrm{mg}$ ), DMT-MM (1.65 mmol, $457 \mathrm{mg})$ in $\mathrm{MeOH}(15 \mathrm{~mL})$ were heated in microwave oven for $25 \mathrm{~min}$. The end of reaction was checked with TLC. The solvent was removed under vacuum to give a crude product. The product was purified by column chromatography.

General Method of Formation of Amide From Substituted Benzoic Acid and 2-phenyethylamine or Piperidine in Presence of DMT-MM Under Thermal Heating

Acid $(1.5 \mathrm{mmol}), 2$-phenylethylamine or piperidine (1.65 mmol), DMT-MM (1.65 mmol, $457 \mathrm{mg}$ ) in $\mathrm{MeOH}$
$(15 \mathrm{~mL})$ were refluxed for $3.5 \mathrm{hr}$. The end of reaction was checked with TLC. The solvent was removed under vacuum to give a crude product. The product was purified by column chromatography.

\section{$N$-Phenethylbenzamide}

Column chromatography: eluent, hexane : $\mathrm{EA}=1: 1, \mathrm{R}_{\mathrm{f}}$ $0.55, \mathrm{mp} 112.4{ }^{\circ} \mathrm{C}\left(113-114{ }^{\circ} \mathrm{C}\right.$ lit. $\left.{ }^{15}\right)$ yield $=88.9 \%$ (thermal reaction, reaction time $3.5 \mathrm{hr}$ ), yield $=99.0 \%$ (microwave irradiation reaction, reaction time $25 \mathrm{~min}$.). ${ }^{1} \mathrm{H}$ NMR: $7.70(10(\mathrm{CH}), \mathrm{m}, 10 \mathrm{H}), 6.14(\mathrm{NH}, \mathrm{s}, 1 \mathrm{H}), 3.73\left(\mathrm{NCH}_{2}, \mathrm{t}\right.$, $2 \mathrm{H}), 2.94\left(\mathrm{NCH}_{2} \underline{\mathrm{CH}}_{2}, \mathrm{t}, 2 \mathrm{H}\right)$

\section{$N$-Phenethyl-4-nitrobenzamide}

Column chromatography: eluent, hexane $: \mathrm{EA}=3: 2, \mathrm{R}_{\mathrm{f}}$ $0.59, \mathrm{mp} 149^{\circ} \mathrm{C}\left(149.5-150.5^{\circ} \mathrm{C}\right.$ lit. $\left.{ }^{15}\right)$ yield $=81.5 \%$ (thermal reaction, reaction time, $3.0 \mathrm{hr}$ ), yield $=92.6 \%$ (microwave irradiation reaction, reaction time $25 \mathrm{~min}$.). ${ }^{1} \mathrm{H}$ NMR: 8.27, 8.25, 7.84, $7.82(4(\mathrm{CH}), \mathrm{dd}, 4 \mathrm{H}), 7.33\left(\mathrm{C}_{6} \mathrm{H}_{5}, \mathrm{~m}, 5 \mathrm{H}\right), 6.13(\mathrm{NH}, \mathrm{s}$, $1 \mathrm{H}), 3.76\left(\mathrm{NCH}_{2}, \mathrm{t}, 2 \mathrm{H}\right), 2.96\left(\mathrm{NCH}_{2} \mathrm{C}_{2}, \mathrm{t}, 2 \mathrm{H}\right)$.

\section{$N$-phenethyl-4-methoxybenzamide}

Column chromatography: eluent, hexane $: \mathrm{EA}=3: 2, \mathrm{R}_{\mathrm{f}}$ $0.64, \mathrm{mp} 118^{\circ} \mathrm{C}\left(117.5-118.5^{\circ} \mathrm{C}\right.$ lit. $\left.{ }^{15}\right)$ yield $=70.8 \%$ (thermal reaction, reaction time, $3.0 \mathrm{hr}$ ), yield $=91.7 \%$ (microwave irradiation reaction, reaction time $25 \mathrm{~min}$.). ${ }^{1} \mathrm{H}$ NMR: 8.27, 8.25, 7.84, 7.82 (4(CH), dd, $4 \mathrm{H}), 7.33\left(\mathrm{C}_{6} \mathrm{H}_{5}, \mathrm{~m}, 5 \mathrm{H}\right), 6.13$ $(\mathrm{NH}, \mathrm{s}, 1 \mathrm{H}), 3.76\left(\mathrm{NCH}_{2}, \mathrm{t}, 2 \mathrm{H}\right), 2.96\left(\mathrm{NCH}_{2} \underline{\mathrm{C}}_{2}, \mathrm{t}, 2 \mathrm{H}\right)$

\section{$N$-Phenethyl-3-methoxybenzamide}

Column chromatography: eluent, hexane : $\mathrm{EA}=3: 2, \mathrm{R}_{\mathrm{f}}$ 0.66 , mp $112-113{ }^{\circ} \mathrm{C}\left(113-114{ }^{\circ} \mathrm{C}\right.$ lit. $\left.{ }^{19}\right)$ yield $=72 \%$ (thermal reaction, reaction time, $3.0 \mathrm{hr}$ ), yield $=92.0 \%$ (microwave irradiation reaction, reaction time $25 \mathrm{~min}$.). ${ }^{1} \mathrm{H}$ NMR: $7.5(9(\mathrm{CH}), \mathrm{m}, 9 \mathrm{H}), 6.10(\mathrm{NH}, \mathrm{s}, 1 \mathrm{H}), 3.82\left(\mathrm{OCH}_{3}, \mathrm{~s}, 3 \mathrm{H}\right)$, $\left.3.66 \mathrm{NCH}_{2}, \mathrm{t}, 2 \mathrm{H}\right) 2.88\left(\mathrm{NCH}_{2} \mathrm{C}_{2}, \mathrm{t}, 2 \mathrm{H}\right)$. 


\section{Phenyl-1-piperadinymethanone}

Column chromatography: eluent, hexane $: \mathrm{EA}=2: 1, \mathrm{R}_{\mathrm{f}}$ 0.30 , yield $=79.3 \%$ (thermal reaction, reaction time, $11.5 \mathrm{hr}$ ), yield $=91.7 \%$ (microwave irradiation reaction, reaction time 30 min.). ${ }^{1} \mathrm{H}$ NMR: 7.34 (5(CH), m, 5H), $3.66\left(\mathrm{NCH}_{2}, \mathrm{~m}\right.$, $\left.2 \mathrm{H}), 3.29\left(\mathrm{NCH}_{2}, \mathrm{~m}, 2 \mathrm{H}\right), 1.62\left(\mathrm{~N}_{\left(\mathrm{CH}_{2}\right.}\right)_{2}\left(\mathrm{CH}_{2}\right)_{2}, \mathrm{~m}, 4 \mathrm{H}\right), 1.46$ $\left(\left(\mathrm{CH}_{2}\right)_{2} \underline{\mathrm{CH}}_{2}, \mathrm{~m}, 2 \mathrm{H}\right)$.

\section{4-Nitrophenyl-1-piperidinylmethanone}

Column chromatography: eluent, hexane $: E A=2: 1, R_{\mathrm{f}}$ 0.23 , yield $=59.6 \%$ (thermal reaction, reaction time, $24 \mathrm{hr}$ ), yield $=75.8 \%$ (microwave irradiation reaction, reaction time $30 \mathrm{~min}$.). ${ }^{1} \mathrm{H}$ NMR: 8.27, 8.20, 7.57, 7.55 (4(CH), dd, 4H), $7.27(\mathrm{NH}, \mathrm{s}, 1 \mathrm{H}), 3.73\left(\mathrm{NCH}_{2}, \mathrm{~m}, 2 \mathrm{H}\right), 3.29\left(\mathrm{NCH}_{2}\right.$, $\mathrm{m}, 2 \mathrm{H}), 1.70\left(\mathrm{~N}\left(\mathrm{CH}_{2}\right)_{2}\left(\mathrm{CH}_{2}\right)_{2}, \mathrm{~m}, 4 \mathrm{H}\right), 1.53\left(\left(\mathrm{CH}_{2}\right)_{2} \mathrm{CH}_{2}\right.$, $\mathrm{m}, 2 \mathrm{H})$.

\section{General Method of Formation of Amides from Sub- stituted Benzoic Acid and Piperidine in in Presence of DCC Under Microwave Irradiation}

Acid (2.5 mmol), piperidine (3 mmol), DCC (3.75 mmol) and pyridine $(0.25 \mathrm{mmol})$ in dry THF $(5 \mathrm{~mL})$ were heated in a microwave oven at $120{ }^{\circ} \mathrm{C}$ (power $60 \mathrm{~W}$ ) for $25 \mathrm{~min}$. The end of reaction was checked with TLC. The solvent was removed under vacuum to give a crude product. The product was purified by column chromatography.

\section{Phenyl-1-piperadinymethanone}

Column chromatography: eluent, hexane $: E A=2: 1, R_{\mathrm{f}}$ 0.30 , yield $=52.8 \%$ (thermal reaction, reaction time, 10 $\mathrm{hr}$ ), yield $=63 \%$ (microwave irradiation reaction, reaction time $25 \mathrm{~min}$.). ${ }^{1} \mathrm{HNMR}: 7.34(5(\mathrm{CH}), \mathrm{m}, 5 \mathrm{H}), 3.66\left(\mathrm{NCH}_{2}, \mathrm{~m}\right.$, $2 \mathrm{H}), 3.29\left(\mathrm{NCH}_{2}, \mathrm{~m}, 2 \mathrm{H}\right), 1.62\left(\mathrm{~N}\left(\mathrm{CH}_{2}\right)_{2}\left(\mathrm{CH}_{2}\right)_{2}, \mathrm{~m}, 4 \mathrm{H}\right)$, $1.46\left(\left(\mathrm{CH}_{2}\right)_{2} \mathrm{C}_{2}, \mathrm{~m}, 2 \mathrm{H}\right)$.

\section{4-Methoxybenzonyl-1-piperadinylmethanone}

Column chromatography: eluent, hexane $: \mathrm{EA}=2: 1, \mathrm{R}_{\mathrm{f}}$ 0.23 , yield $=50.4 \%$ (thermal reaction, reaction time $12 \mathrm{hr}$ ), yield $=60 \%$ (microwave irradiation reaction, reaction time 25 min.) ${ }^{1} \mathrm{HNMR}: 7.37,7.35,6.91,6.89$ (4(CH), dd, 4H), $3.82\left(\mathrm{OCH}_{3}, \mathrm{~s}, 3 \mathrm{H}\right), 3.45\left(\mathrm{~N}\left(\mathrm{CH}_{2}\right)_{2}, \mathrm{~m}, 4 \mathrm{H}\right), 1.67\left(\mathrm{~N}\left(\mathrm{CH}_{2}\right)_{2}\right.$ $\left.\left(\mathrm{C}_{2}\right)_{2}, \mathrm{~m}, 4 \mathrm{H}\right), 1.59\left(\left(\mathrm{CH}_{2}\right)_{2} \mathrm{C}_{2}, \mathrm{~m}, 2 \mathrm{H}\right)$.

\section{REFERENCES}

1. Glynn, D.; Berier, D.; Woodward, S. Teterahedron Lett. 2008, 49, 5687.

2. Montalbetti, C. A. G. N.; Falque, V. Tetrahedron 2005, $61,1082$.

3. Kishikawa, K; Yamamoto M.; Kohmoto, S.; Yamada, K. Synth. Commun. 1989, 993.

4. Munakami, M.; Hayashi, M.; Tamura, N.; Hishino, Y.; Ito Y. Teterahedron Lett. 1996, 37, 7541.

5. Chandrasekhar, S.; Mohamede, T.; Uma, G. Teterahedron Lett. 1997, 38, 8089.

6. Kamninski, Z. J.; Paneth, P.; Rudzinski, J. J. Org. Chem. 1998, 63, 4248.

7. Bailen, M.; Chichilla, R.; Dodsworth, D. J.; Najera, C. J. Org. Chem. 1999, 64, 8936.

8. van Leeuwen, S. H.; Quaedflieg, P. J. L. M.; Broxterman, Q. B.; Liskamp, R. M. J. Teterahedron Lett. 2002, 43, 9203.

9. Quelever, G.; Burlet, S.; Garino, C.; Pietrancosa, N.; Laras, Y.; Kraus, J.-L. J. Comb. Chem. 2004, 6, 695.

10. Wei, Z.; Yimin, L. QSAR \& Comb. Sci. 2006, 25, 724.

11. Valeur, E.; Bradley, M. Tetrahedron 2007, 63, 8855.

12. De Wael, K.; Buschop, H.; De Smet, L.; Adriaens, A. Talanta 2008, 76, 309.

13. Kunishima, M.; Kawachi, C.; Morita, J.; Terao, K.; Iwasaki, F.; Tani, S.; Tetrahedron 1999, 55, 13159.

14. Kunishima, M.; Kawachi, C.; Iwasaki, F.; Terao, K.; Tani, S.; Tetrahedron Lett. 1999, 40, 5327.

15. Kunishima, M.; Kawachi, C.; Hioki, K.; Terao, K.; Tani, S.; Tetrahedron 2001, 57, 1551.

16. Caddick, S. Tetrahedron 1995, 51, 10403.

17. Lidstrom, P.; Tierney, J.; Wathey, B.; Westman, J. Tetrahedron 2001, 57, 9225.

18. Kappe, C. O. Angew. Chem., Int. Ed. 2004, 43, 6250.

19. Kunishima, M.; Kawachi, C.; Morita J.; Terao, K.; Iwasaki, F.; Tani, S. Tetrahedron 1999, 55, 13159. 